\title{
Lanczos spintensor in Gödel spacetime via factorization of the metric tensor
}

\section{Espintensor de Lanczos en el espacio-tiempo de Gödel a través de la factorización del tensor métrico}

\author{
M. Shadab+(D), J. López-Bonilla*1] and G. Sánchez-Meléndez* \\ ${ }^{+}$Department of Natural and Applied Sciences, School of Science and \\ Technology, Glocal University, Saharanpur 247121, India \\ * ESIME-Zacatenco, Instituto Politécnico Nacional, Edif. 4, 1er. Piso, \\ Col. Lindavista, C.P. 07738, CDMX, México
}

\begin{abstract}
We show a factorization of the metric tensor in Gödel cosmological model which gives a generator for the Lanczos spintensor of the conformal tensor.

Keywords. Gödel geometry; Lanczos potential; Weyl tensor.

Resumen. Mostramos una factorización del tensor métrico en el modelo cosmológico de Gödel, lo cual da un generador para el espíntensor de Lanczos del tensor conformal.

Palabras Clave. Geometría de Gödel; Potencial de Lanczos; Tensor de Weyl.

How to Cite. M. Shadab, J. Lopez-Bonilla and G. Sanchez-Melendez, "Lanczos spintensor in Gödel spacetime via factorization of the metric tensor", Jou. Cie. Ing., vol. 13, no. 1, pp. 24-27, 2021. doi $10.46571 / \mathrm{JCl} .2021 .1 .4$
\end{abstract}

Received: 10/03/2021 Revised: 02/05/2021 Accepted: 28/05/2021

The Lanczos potential $K_{a b c}$ has the properties [1-3]:

$$
K_{a b c}=-K_{b a c}, \quad K_{a b c}+K_{b c a}+K_{c a b}=0, \quad K_{a b}^{b}=0, \quad K_{; c}^{a b c}=0,
$$

and it permits to construct the conformal tensor in according with the fundamental relation [1]:

$$
C_{a b c d}=K_{a b c ; d}-K_{a b d ; c}+K_{c d a ; b}-K_{c d b ; a}+K_{a d} g_{b c}-K_{a c} g_{b d}+K_{b c} g_{a d}-K_{b d} g_{a c},
$$

1 Corresponding author: joseluis.lopezbonilla@gmail.com 
where:

$$
K^{a b}=K^{b a}=K_{; c}^{a c b} .
$$

For the Gödel's geometry [4 7$]$ :

$$
d s^{2}=\left(d x^{0}\right)^{2}+2 e^{x^{3}} d x^{0} d x^{1}+\frac{1}{2} e^{2 x^{3}}\left(d x^{1}\right)^{2}-\left(d x^{2}\right)^{2}-\left(d x^{3}\right)^{2},
$$

we can write the following factorization of the metric tensor:

$$
\left(g^{a b}\right)=\left(\begin{array}{cccc}
-1 & 2 e^{-x^{3}} & 0 & 0 \\
2 e^{-x^{3}} & -2 e^{-2 x^{3}} & 0 & 0 \\
0 & 0 & -1 & 0 \\
0 & 0 & 0 & -1
\end{array}\right)=\left(P^{a c} Q_{c}^{b}\right)
$$

such that:

$$
\left(P^{a c}\right)=\left(\frac{1}{9} E^{a c}\right)=\frac{1}{9} \operatorname{Diag}\left(-\frac{3}{4}, \frac{1}{4}, \frac{1}{4}, \frac{1}{4}\right), \quad\left(Q_{c}^{b}\right)=9\left(\begin{array}{cccc}
\frac{4}{3} & -\frac{8}{3} & 0 & 0 \\
8 e^{-x^{3}} & -8 e^{-2 x^{3}} & 0 & 0 \\
0 & 0 & -4 & 0 \\
0 & 0 & 0 & -4
\end{array}\right)
$$

where $E^{a c}$ is the Ricci tensor without trace. It is surprising that the factor $P^{a c}$ generates the Lanczos potential for this Gödel cosmological model:

$$
K_{a b c}=P_{c b ; a}-P_{c a ; b}=\frac{1}{9}\left(R_{c b ; a}-R_{c a ; b}\right), \quad\left(R_{a b}\right)=\left(\begin{array}{cccc}
-1 & -e^{x^{3}} & 0 & 0 \\
-e^{x^{3}} & -e^{2 x^{3}} & 0 & 0 \\
0 & 0 & 0 & 0 \\
0 & 0 & 0 & 0
\end{array}\right), \quad R=R_{a}^{a}=-1
$$

thus (2), (3) and (7) imply the following components different to zero:

$$
\begin{array}{llrl}
K_{031}=K_{103}=\frac{1}{2} K_{130} & =\frac{1}{18} e^{x^{3}}, & K_{131} & =\frac{1}{6} e^{2 x^{3}} \\
-\frac{1}{2} K^{0}{ }_{0}=K^{1}{ }_{1}=K^{3}{ }_{3}=\frac{1}{6}, & K^{0}{ }_{1}=-\frac{1}{2} e^{x^{3}} .
\end{array}
$$

$C_{0303}=-C_{2323}=-\frac{1}{2} C_{0202}=\frac{1}{6}, C_{0313}=\frac{1}{2} C_{1220}=\frac{1}{6} e^{x^{3}}, C_{0101}=\frac{1}{4} C_{1313}=-\frac{1}{5} C_{1212}=\frac{1}{12} e^{2 x^{3}}$.

Besides, it is possible to show the relation:

$$
K^{a b c}=-\frac{2}{9} C_{; r}^{a b c r}
$$

which may be considered as the inversion of (2), that is, the Weyl tensor generates the Lanczos potential in Gödel spacetime. It is possible to deduce the wave equation [8] for some quantities of interest, for example:

$$
\square K^{a b c} \equiv K_{; r}^{a b c ; r}=-3 K^{a b c}, \quad \square R_{a b}=2 \square K_{a b}=-6 K_{a b},
$$


and several identities involving the Einstein, Ricci and Riemann tensors:

$$
\begin{array}{cr}
K_{a b ; c}+K_{b c ; a}+K_{c a ; b}=0, \quad K_{; b}^{a b}=0, & R_{a b c r} K^{a b c}=0, \\
R_{[a}^{r} K_{b] c r}-R_{c}^{r} K_{a b r}-R_{q}^{r} K_{r[a}^{q} g_{b] c}=K_{a b c}, & K_{a b}=\frac{1}{6}\left(R_{a b}-2 R_{a c r b} G^{c r}\right),
\end{array}
$$

and $K_{a b}$ turns out to be a potential for the Lanczos tensor:

$$
K_{a b c}=-\frac{2}{9}\left(K_{c a ; b}-K_{c b ; a}\right),
$$

thus (12) is the inversion of (3). We indicate the following non-zero components:

$$
\begin{gathered}
\Gamma_{013}=\Gamma_{103}=-\Gamma_{301}=\frac{1}{2} e^{x^{3}}, \Gamma_{113}=-\Gamma_{311}=\frac{1}{2} e^{2 x^{3}}, \Gamma_{01}^{3}=\Gamma_{13}^{0}=\frac{1}{2} e^{x^{3}}, \Gamma_{03}^{0}=1, \Gamma_{03}^{1}=-e^{-x^{3}}, \\
\Gamma_{11}^{3}=\frac{1}{2} e^{2 x^{3}}, \quad R_{0303}=\frac{1}{2}, \quad R_{0313}=\frac{1}{2} e^{x^{3}}, \quad R_{1313}=3 R_{0101}=\frac{3}{4} e^{2 x^{3}}, \quad(13) \\
\left(G^{a}{ }_{c}\right)=\left(\begin{array}{cccc}
-\frac{1}{2} & -e^{x^{3}} & 0 & 0 \\
0 & \frac{1}{2} & 0 & 0 \\
0 & 0 & \frac{1}{2} & 0 \\
0 & 0 & 0 & \frac{1}{2}
\end{array}\right) .
\end{gathered}
$$

An adequate linear combination of $R_{a b}$ and $K_{a c}$ gives an interesting generator:

$$
\begin{aligned}
K_{a b c} & =-\frac{\sqrt{2}}{18}\left(B_{c a ; b}-B_{c b ; a}\right), & B_{; c}^{a c} & =0, \\
B_{a c} & =\frac{1}{\sqrt{2}}\left(6 K_{a c}-R_{a c}\right), & \square B_{a c} & =-6 \sqrt{2} K_{a c},
\end{aligned}
$$

and it is remarkable the fulfillment of the Gauss equation $7,9,11$ :

$$
R_{a b c r}=B_{a c} B_{b r}-B_{a r} B_{b c}, \quad B_{a c}=-\sqrt{2} R_{a b r c} G^{b r}, \quad \stackrel{*}{\square} R^{* a b c r} R_{a b c r}=0,
$$

hence $B_{a c}$ generates the Lanczos potential via the differential relation (14) and also the curvature tensor through the algebraic expression (15).

The idea of factoring the metric tensor, as in (5), to obtain a generator of the Lanczos potential, also works in Kerr spacetime [12].

\section{References}

[1] C. Lanczos, "The splitting of the Riemann tensor," Rev. Mod. Phys., vol. 34, no. 3, pp. 379-389, 1962.

[2] J. D. Zund, "Sur le spineur de Lanczos en relativité générale, Comptes Rendus Acad. Sci. (Paris), no. A276, pp. 1629-1631, 1973.

[3] Z. Ahsan, The potential of fields in Einstein's theory of gravitation, Springer, Singapore, 2019.

[4] K. Gödel, "An example of a new type of cosmological solution of Einstein's field equations of gravitation," Rev. Mod. Phys., vol. 21, no. 3, pp. 447-450, 1949.

[5] S. Chandrasekhar, J. P. Wright, "The geodesics in Gödel's Universe," Proc. Nat. Acad. Sci. USA, no. 47, pp. 341-347, 1961.

[6] J. L. Synge, Relativity: the general theory, North-Holland, Amsterdam, 1976. 
[7] H. Stephani, D. Kramer, M. MacCallum, C. Hoenselaers, E. Herlt, "Exact solutions of Einstein's field equations, Cambridge University Press, 2003.

[8] Z. Ahsan, J. López-Bonilla, A. Rangel-Merino, "Wave equation in Gödel model," J. Vect. Rel., vol. 4, no. 2, pp. 80-88, 2009

[9] H. Rund, D. Lovelock, Tensors, differential forms, and variational principles: Dover, New York, 1989.

[10] G. González, J. López-Bonilla, M. Rosales, "An identity for $R_{n}$ embedded into $E_{n+1}$," Pramana J. Phys., vol. 42, no. 2, pp. 85-88, 1994.

[11] P. Lam-Estrada, J. López-Bonilla y H. E. Caicedo-Ortiz, "Gödel spacetime and Lanczos potential," Jou.Cie.Ing., vol. 12, no. 1, pp. 124-126, 2020. doi 10.46571/JCI.2020.1.11.

[12] J. López-Bonilla, M. Shadab, S. Ray, "Factorization of the metric tensor in Kerr geometry," Scientific Voyage, vol. 2, no. 2, 2021 to appear.

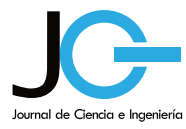

